\title{
Improving educational efficiency
}

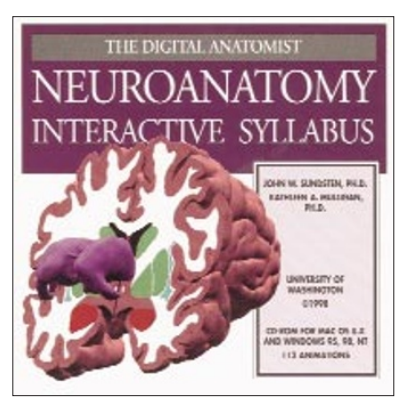

\section{The Neuroanatomy Interactive Syllabus}

by John Sundsten and Kathleen Mulligan

University of Washington, Seattle, 1999. $\$ 200$

CD-ROM

Reviewed by Stewart Hendry

The costs of postgraduate education continue to increase with an inevitability previously observed only for death and taxes. The best reason given for this state of affairs is that we who educate are inefficient, particularly in failing to take advantage of revolutions in data storage and transfer. When I compare the means by which the best in the business educated me in the ways of the brain and how I attempt to pass along their wisdom, I would have to agree. Nothing much has changed in twenty years.

The Neuroanatomy Interactive Syllabus by John Sundsten and Kathleen Mulligan is one of several available CDROMs that attempts to initiate those changes. In a well-constructed and easily navigated format, Drs. Sundsten and Mulligan provide a rich list of topics that are explored thoroughly. Whether the topic is a particular integrated system, an individual level of the central nervous system or a specific issue, the authors have done a creditable job of providing both breadth and depth. To take one early example, the chapter that deals with CNS development effectively mixes the embryology of the brain and spinal cord with the molecular biology of pattern formation. The former provides a beginning student with the simplest plan of CNS organization, upon which he or she can build a knowledge of details, whereas the latter permits the student to make sense

Stewart Hendry is at the Zanvyl Krieger Institute, Johns Hopkins University email: HENDRYS@jhuvms.hcf.jhu.edu of how a simple tube becomes a human brain.

Some 21 topics are covered in total, with particularly close attention paid to sensory systems. Thus, the somatosensory system is divided into separate topics that comprise the lemnis-

cal and spinothalamic components. Such a division is wise, as experience indicates that students are most comfortable dealing with the two as distinct functional paths. For these and all other topics, judicious use is made of animation to provide the student with an excellent three-dimensional view of each part in an integrated system. Perhaps this is where the Neuroanatomy

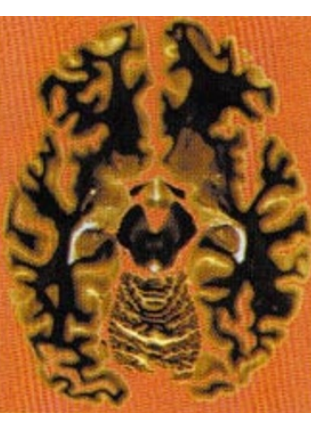
Interactive Syllabus is strongest, as it gives what most beginning students desire but the artistically impaired faculty member has singular difficulty in providing. So numerous are the animated presentations, I would have to say that wherever a compelling three-dimensional view is necessary, this CD-ROM is there to give it.

The layout of each section is exemplary, as the authors take pains to introduce a topic, examine it in overview, explore it in detail and question the student on knowledge accrued. It is true, however, that the last of these, which comprise a short quiz of each image, is somewhat limited because even when topics of function (for example, lateral gaze) are presented, the questions deal only with identification of structures. The overall layout provides a beginning student with the opportunity to add to his or her understanding at a speed and depth determined by the student rather than by the instructor.

Because this is a CD-ROM that deals with neuroanatomy, the authors have decided to use slices of unfixed, unstained human brains to document the locations of nuclei and tracks. One could quibble about resolution or magnification of some slices, but there is no doubt that the great majority of what needs to be shown is readily apparent to the viewer. The authors also make good use of magnetic resonance images, displayed in the three standard planes, as a means to introduce the future clinicians in their audience to the images they will use in practice. Well represented throughout are stained sections of human material, particular of basal forebrain, thalamus and brainstem. For all sections and images, a simple click on the position of a structure in question provides the viewer with its name. Accompanying each figure is text, sequestered in a narrow box. That my eyes tended to avoid these words is largely to the authors' credit, as the treatment of subjects was so efficient and accurate it demanded little attention from a reviewer. For a beginning student, however, the text will likely be a point of great interest, particularly when an individual topic is examined for the second or third time.

How does all this speak to the question of efficiency? The Neuroanatomy Interactive Syllabus and other CD-ROMS of its kind take a traditional approach to teaching the organization of the CNS; in that way, they resemble first-year classes on the subject taught at most major medical schools. Were students to use such a disk, and there is every good reason they should, many of the questions and much of the time given over to painfully basic facts could be used for explanation of more enlightening concepts. As such, any student at the start of a biomedical education would benefit from the acquisition and use of this CD-ROM as a means to lay straight a path that the faculty could either pave or pit, depending on its own predilections. 